\title{
(2) OPEN ACCESS \\ Vaccination and multiple sclerosis in the era of the COVID-19 pandemic
}

\author{
Tobias Monschein 다, ${ }^{1}$ Hans-Peter Hartung ${ }^{1,2}$ Tobias Zrzavy (1) , \\ Michael Barnett $\odot{ }^{3}{ }^{3}$ Nina Boxberger, ${ }^{4}$ Thomas Berger, ${ }^{1}$ Jeremy Chataway, ${ }^{5}$ \\ Amit Bar-Or, ${ }^{6}$ Paulus Stefan Rommer ${ }^{0}, 1^{1,4}$ Uwe K. Zett $^{4}$
}

\begin{abstract}
- Additional supplemental material is published online only. To view, please visit the journal online (http://dx. doi.org/10.1136/jnnp-2021326839).
\end{abstract}

For numbered affiliations see end of article.

\section{Correspondence to} Dr Tobias Monschein, Department of Neurology, Medical University of Vienna, Vienna, Austria; tobias. monschein@meduniwien.ac. at and Professor Hans-Peter Hartung, Department of Neurology, Universitätsklinikum Düsseldorf, Medical Faculty, Heinrich Heine University, Düsseldorf, Germany; hanspeter.hartung@uni-duesseldorf. de

TM and H-PH contributed equally.

Received 11 April 2021 Accepted 16 June 2021 Published Online First 5 August 2021

Check for updates

(c) Author(s) (or their employer(s)) 2021. Re-use permitted under CC BY-NC. No commercial re-use. See rights and permissions. Published by BMJ.

To cite: Monschein $T_{\text {, }}$ Hartung H-P, Zrzavy T, et al. J Neurol Neurosurg Psychiatry 2021:92:1033-1043.

\section{INTRODUCTION}

In the last century, several pandemics were caused by various influenza virus subtypes, with the Spanish influenza of 1918-1920 being the most severe. ${ }^{1}$ Coronaviruses were first described in humans in the 1960s, and since then seven human pathogenic coronaviruses have been described. ${ }^{2}$ The first severe outbreak was in 2002 with the severe acute respiratory syndrome coronavirus (SARS-CoV), followed by the Middle East respiratory syndrome coronavirus (MERS-CoV) outbreak in 2012. ${ }^{3}$ The current COVID-19 pandemic, which broke out in Wuhan, China, in late 2019, is caused by the highly transmissible RNA virus SARS-CoV-2. ${ }^{4}$ As of 11 June 2021, 174439909 COVID-19 cases and 3768987 deaths have been reported worldwide, according to the WHO Dashboard. ${ }^{5}$

Infectious diseases significantly contribute to morbidity in patients with multiple sclerosis (MS), since the risk of suffering from an infection is increased by MS per se and may be further enhanced by disease-modifying therapies (DMTs) and frequent comorbidities. ${ }^{6-9}$ As a result, infectious diseases may heighten the risk of disease progression in MS and result in reduced life expectancy. ${ }^{10-12}$ The COVID-19 pandemic, in particular, presents new challenges for patients with MS. Since the approval of messenger RNA (mRNA) and non-replicating vector coronavirus vaccines by the European Medicines Agency (EMA), the UK Medicines and Healthcare products Regulatory Agency (MHRA), the US Food and Drug Administration (FDA), Health Canada (HC) and the Australian Therapeutic Goods Administration (TGA), neurologists are facing an unprecedented demand for information on vaccination in MS, particularly in the context of DMTs. ${ }^{13-17}$ This review aims to provide a clinical practice-oriented approach to SARS-CoV-2 vaccination in MS in the era of the COVID-19 pandemic. ${ }^{18-21}$

\section{COVID-19}

COVID-19 is a disease caused by the highly contagious RNA virus SARS-CoV-2. It shares a 79\% genetic match with SARS-CoV and a $50 \%$ genetic match with MERS-CoV. ${ }^{22}$ Based on phylogenetic studies, the presumed transmission from animals to humans probably originates from bats and the pangolin. ${ }^{23}$ After a naso-oral infection route and similar to SARS-CoV, SARS-CoV-2 binds to ACE-2 to enter cells, with the latter exhibiting a higher binding affinity. ${ }^{24}$ On entering the respiratory epithelial cells, SARS-CoV-2 starts replicating and spreading rapidly until it reaches the alveolar epithelial cells of the lung. Hence, a robust innate immune response is triggered. ${ }^{25}$ The spike protein is recognised by pattern recognition receptors and leads, via downstream signalling, to increased interferon production by innate immune cells. Antigen-specific adaptive immunity is critical for the immune response to SARS-CoV-2 infection. Both CD8 and CD4 $\mathrm{T}$ cells are activated via their respective major histocompatibility complexes I or II, directly targeting virus-infected cells, as well as stimulating other immune cells such as B cells and macrophages. ${ }^{26} 27$ Thereby, in contrast to neutralising antibody titres, SARS-CoV-2-specific CD4 and CD8 cells are associated with a milder disease course, suggestive of protective immunity. ${ }^{28-31}$

Nevertheless, the production of neutralising antibodies seems to be crucial in the course of the adaptive immune response, as these prevent the infection of further cells with the virus, in contrast to the cellular immune response, which eliminates already infected cells. ${ }^{32}$ Depending on the severity of the infection, a rapid spread along with tissue destruction can lead to cytokine storm syndrome (CSS), which can eventually progress to acute respiratory distress syndrome (ARDS). ${ }^{34-36}$

Age and comorbidities are the main risk factors for the development of ARDS and thus for a fatal outcome. $^{37}$ Among other cytokines, interleukin 6 (IL-6) is thought to play a central role in CSS and ARDS pathogenesis, as emphasised by high IL-6 levels in patients with respiratory failure. ${ }^{38} 39$ Based on these findings, a clinical phase III trial with a humanised monoclonal antibody against the IL- 6 receptor was initiated; amelioration of a severe COVID-19 disease course, but no improvement in overall survival, was demonstrated. ${ }^{40} s 41-s 43$ Furthermore, treatment with convalescent plasma has been examined. However, so far there are only reliable data on using high-titre convalescent serum in older patients with early onset ( $<72$ hours) and mild symptoms, but it could also be considered for younger high-risk patients (and certain immunodeficient patients) with COVID-19. ${ }^{54, s 45}$ In addition, SARS-CoV-2 neutralising antibodies (casirivimab and imdevimab, REGN-COV2) received emergency approval from the FDA (21 November 2020) and the EMA (26 February 2021) for treatment of COVID-19. ${ }^{46, s 47}$ Moreover, dexamethasone treatment reduced 28-day mortality in severe COVID-19 
in the RECOVERY study, prompting a strong recommendation from the WHO to use this corticosteroid in patients with severe COVID $-19 .^{\text {s48,s49 }}$

The most frequent symptoms of COVID-19 include fever, dry cough and fatigue, and less commonly diarrhoea. ${ }^{550}$ Hyposmia/ anosmia and dysgeusia/ageusia may also occur. ${ }^{551}$ Based on these clinical findings, migration of the virus to the CNS (central nervous system) via axonal transport and/or blood-brain barrier transmigration has been hypothesised. ${ }^{552}$ Possible additional neurological manifestations of COVID-19, based on case reports or small series, can be classified into five classes: (1) encephalopathy, (2) inflammatory CNS syndromes such as encephalitis or ADEM (acute disseminated encephalomyelitis), (3) ischaemic strokes, (4) peripheral nerve involvement such as Guillain-Barre

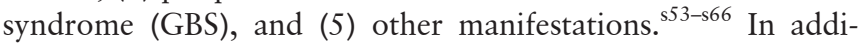
tion, Nalbandian et al described postacute COVID-19 syndrome developing 4 or more weeks after infection, which in up to $38 \%$ of cases manifests itself with fatigue, headaches, dysautonomia and cognitive impairment. ${ }^{567}$ Significant neurological and psychiatric morbidity 6 months after SARS-CoV-2 infection was noted in $33.6 \%$ of individuals in a large retrospective observational study using data of 236379 patients from the US TriNetX network. ${ }^{\text {s68 }}$

\section{COVID-19 AND MS}

Currently, there is no evidence of a generally heightened risk of SARS-CoV-2 infection or a more severe course in patients with MS. ${ }^{69}$ Data regarding patients with NMOSD (neuromyelitis optica spectrum disorders) are controversial. ${ }^{\text {s70,s71 }}$ Prognostic factors associated with a more severe COVID-19 in people with MS are older age, higher disability (EDSS [expanded disability status scale]), obesity, cardiovascular comorbidities, progressive disease course and prior corticosteroid treatment. ${ }^{\text {s2-s75 }}$ Exposure to DMTs per se does not appear to convey an elevated risk of developing more severe COVID-19.

Therefore, according to the MS International Federation (MSIF), patients with MS should continue their DMT and follow the general WHO recommendations regarding COVID-19 unless otherwise recommended by the treating neurologist.

Based on the currently available data, there seems to be no overall elevated risk for patients with MS using a DMT. There is evidence that interferon- $\beta$ is associated with a lower COVID19 -related hospitalisation rate. ${ }^{\mathrm{s} 76}$ However, some reports suggest a higher risk of severe COVID-19 illness in patients receiving a $\mathrm{B}$ cell-depleting agent such as ocrelizumab or rituximab. ${ }^{\text {s77-s79 }}$ These data are preliminary and B cell-depleting therapies have no pathophysiological association with ARDS or hypercoagulopathy, the most critical factors for the development of severe COVID-19. ${ }^{\text {s74,s79-s82 }}$ On the other hand, continuous CD20 depletion is associated with lower levels of IgM and, to a lesser extent, IgG hypogammaglobulinaemia and the risk of severe infection, and thus could easily contribute to a more severe course of infection. ${ }^{\mathrm{s} 60, \mathrm{~s} 61}$ Protective cross-reactive immunity to a previous 'conventional' coronavirus infection may be lowered.

Taking currently available evidence together, patients with MS should stay on their DMTs during the pandemic. ${ }^{\text {s8-s88 }}$ Depending on the individual situation, if cyclical therapy (rituximab, ocrelizumab, alemtuzumab or cladribine) is used, postponement of the next dose can be considered as this may enable a better protective vaccine response. ${ }^{899-594}$ Age and comorbidities are probably more critical than DMTs for risk stratification in COVID-19. ${ }^{595}$

\section{COVID-19, VACCINATION AND MS}

From the first variolated smallpox vaccine by English physician Edward Jenner in 1796 to the era of gene-based vaccines, there are now over 70 approved vaccines available. ${ }^{596}$ Their effectiveness has been recently comprehensively reviewed by Nabel, who calculated that tens of millions of people died from nowpreventable infections before vaccination was introduced in the 20th century ( $>17$ million deaths from diphtheria alone). ${ }^{\mathrm{s} 96}$

Vaccines can be classified into live/attenuated, non-live/inactivated and gene-based. The principle of live vaccines is based on the attenuation of the virus or its ability to replicate through genetic modification. This can be achieved, for example, by optimal growth at a lower temperature (cold-adapted). ${ }^{597,598}$ Live vaccines generate a strong immunity; however, they are associated with risk of symptomatic disease, especially in immunocompromised individuals. ${ }^{\text {s99 }}$

Inactivated vaccines (subsuming inactivation, subunit, split vaccine, conjugate vaccine) have the advantage of not causing infection since only an inactivated pathogen or components thereof are administered, but at the sacrifice of lower immunogenicity and shorter immunity. ${ }^{\text {s100 }}$

Adjuvants, components that trigger an enhanced antigenspecific immune response, are therefore often used to stimulate the immune response and encompass aluminium salts, lipid A analogues or emulsions. ${ }^{\text {s101 }}$

The third category are gene-based vaccines, including vector vaccines and mRNA vaccines. ${ }^{s 102, s 103}$ Vector vaccines are typically based on an engineered 'vector' virus that has been genetically modified to express, for example, the SARS-CoV-2 spike protein, and is prevented from replicating in vivo. ${ }^{\text {s104 }}$ In the case of the 'Gam-COVID-Vac', also known as the Sputnik V vaccine, two recombinant non-replicating human adenoviruses were used (two for minimising immune response against vector components), and in the case of the 'ChAdOx1 nCoV-19' an unreplicable chimpanzee adenovirus is used. ${ }^{\mathrm{s} 105, \mathrm{~s} 106}$

On the other hand, mRNA vaccines provide the genetic information, in the form of mRNA, for the biosynthesis of a specific antigen. This is administered together with a stabilising reagent (eg, protamine) in a lipid coat to prevent degradation before reaching the cytosol. There a stable protein is synthesised through ribosomal translation and post-translational modification, which then passes to the cell surface and can thus trigger an immune reaction. ${ }^{\text {s107,s108 }}$ Multiple SARS-CoV-2 vaccine types are under development, including inactivated vaccines, recombinant subunit vaccines, nucleic acid vaccines, adenovirus-based vector vaccines and one live-attenuated vaccine (phase I). ${ }^{\text {s109 }}$ Figure 1 provides an overview of the immunology of SARS-CoV-2 vaccination and the mechanisms of DMTs.

Approved vaccines are considered overall relatively safe. The vast majority of adverse events (AE) are limited to local reactions and mild systemic reactions such as fever. Raised body temperature could trigger Uhthoff's phenomenon. ${ }^{\text {s91,s110 }}$ Serious side effects are very rare, of which anaphylaxis is still the most common, with a risk of 0.65 cases per million doses. ${ }^{\text {s111 }}$ Other examples of AEs include vaccine-associated paralytic poliomyelitis with the (live-attenuated) oral polio vaccine, which is sporadic and rare. ${ }^{\text {s112 }}$ This vaccine is now only used in a few developing countries worldwide and is no longer licensed in Europe since 1998 and in the USA since 2000, as a safer, although more expensive, inactivated vaccine is available. ${ }^{\mathrm{s} 113, \mathrm{~s} 114}$ The AS03 adjuvanted influenza A (H1N1) pdm09 vaccine was associated with an increased rate of narcolepsy (according to the Paul Ehrlich Institute, 86 suspected cases in over 30 million 


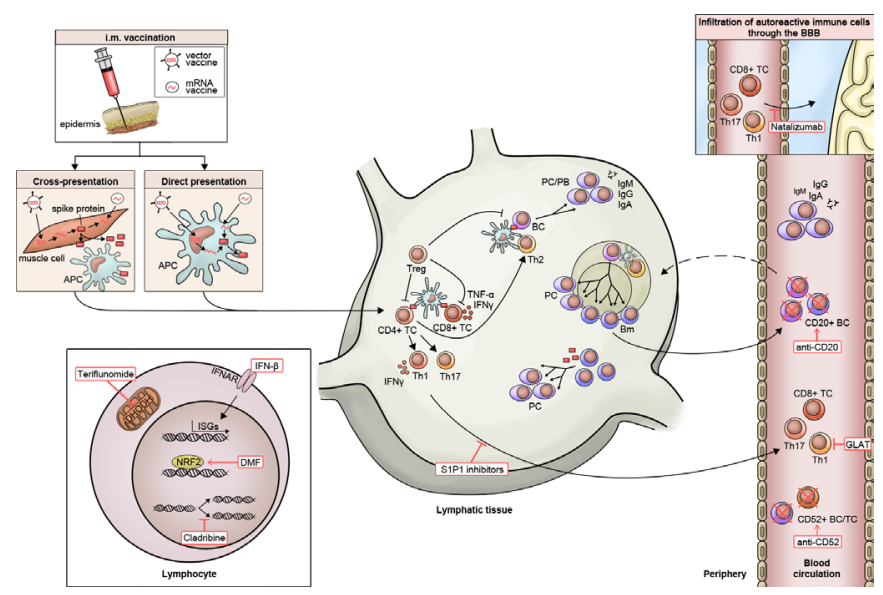

Figure 1 Immunology of SARS-CoV-2 vaccination and the mechanisms of DMTs.

(1) Injection of vaccine into the muscle tissue (1) leading to direct/ cross-presentation of synthesised spike protein to lymphocytes via APC within the lymphatic tissue and (2) providing costimulatory signals for the differentiation of naïve T cells into immune effector cells; antigen recognition/presentation to $B$ cells leads to their activation and migration towards the T-B cell border of the lymph node, where they can subsequently receive additional stimuli by activated T helper (Tn) cells. (3) Affinity maturation and isotype switch result in a sustained production of high-affinity antibodies by predominantly plasma cells but also memory B cells partly emigrating into the circulation. (2) (1) Teriflunomide inhibiting DHODH leading to reduced proliferation of stimulated lymphocytes. (2) Cladribine disrupting DNA synthesis and repair targeting predominantly B cells and T cells. (3) DMF activation and stabilisation of NRF2. (4) IFN-B activating ISGs subsequently decreasing the expression of proinflammatory cytokines. (5) S1P1 preventing egress of T cells from lymph nodes. (6) AntiCD20 antibody depletion of CD20 expressing B cells (eg, memory B cells) and double-positive T cells while sparing plasma cells. (7) Natalizumab binding to the adhesion molecule alpha-4 integrin, thereby reducing cell immigration into the brain via the blood-brain barrier (BBB). (8) GLAT structurally similar to myelin basic protein, preventing $T$ helper cell type 1 (Th1) activation against myelin and promoting activation of $T$ helper cell type 2 (Th2); B cell (BC); plasma cell (PC), plasma blast (PB), memory $B$ cell (BM); IFN- $\beta$ (Interferon- $\beta$ ), NFAR (Interferon- $\alpha / \beta$ receptor), IFN-y (Interferon-y); T cell (TC), T regulatory cell (Treg); tumour necrosis factor alpha (TNF- $\alpha$ ). APC, antigen presenting cells; DHODH, dihydro-orotate dehydrogenase; DMF, dimethyl fumarate; DMTs, disease-modifying therapies; GLAT, glatiramer acetate; ; ISGs, interferon-stimulated genes; mRNA, messenger RNA; NRF2, nuclear factor (erythroid-derived 2)-like 2; S1P1, sphingosine-1-phosphate-1 receptor modulators.

administered vaccinations). ${ }^{s 115, s 116}$ It should be mentioned that the approval by the EMA at that time in the course of swine influenza was based on six studies with a total of 610 subjects, making it impossible to detect very rare AEs. ${ }^{s 117}$ This is also the reason why the phase III trials for the new SARS-CoV-2 vaccines enrolled markedly more participants (eg, 'BNT162b2'—43 448 subjects, of whom 27720 received vaccination) to be able to better detect rare AEs. ${ }^{18}$

Therefore, it can be assumed that very rare side effects were also identifiable in the phase III studies. In addition, particularly in the pivotal trial of the 'mRNA-1273' vaccine, patients with risk factors were also enrolled, including immunosuppressed patients with HIV infection. ${ }^{19}$

According to the WHO, there are currently 287 SARS-CoV-2 vaccines under development, 185 in preclinical phase, 102 in clinical phase, 18 in phase III stage and 5 in phase IV stage (as of 11 June 2021). ${ }^{\text {s118-s120 }}$ Out of 17 vaccines approved recently worldwide, two mRNA vaccines (BNT162b2, 21 December 2020; mRNA-1273, 6 January 2021) and two non-replicating vector virus vaccines (ChAdOx1 nCoV-19, 29 January 2021; 'Ad26.COV2.S', 11 March 2021) have already been licensed by the EMA; two mRNA vaccines (BNT162b2, 2 December 2020; mRNA-1273, 8 January 2021) and the non-replicating vector virus vaccine ChAdOx1 nCoV-19 (30 December 2020) by the MHRA; two mRNA vaccines (BNT162b2, 11 December 2020; mRNA-1273, 18 December 2020) and the non-replicating vector virus vaccine Ad26.COV2.S (27 February 2021) by the FDA; two mRNA vaccines (BNT162b2, 9 December 2020; mRNA-1273, 23 December 2020) and the two non-replicating vector virus vaccines (ChAdOx1 nCoV-19, 26 February 2021; Ad26.COV2.S, 5 March 2021) by HC; and the mRNA vaccine BNT162b2 (25 January 2021) and the non-replicating vector virus vaccine ChAdOx1 nCoV-19 (16 February 2021) by the TGA. ${ }^{16-19}$ s105,s121,s122 In addition, the non-replicating vector virus vaccines 'Gam-COVID-Vac=Sputnik V' in Russia, 'Covaxin (BBV152)' and 'Serum Institute of India - Covishield' in India (same formulation as ChAdOx1 nCoV-19), as well as 'CanSino: Ad5-nCoV' in

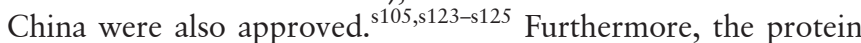
subunit vaccines 'Anhui Zhifei Longcom - RBD-Dimer' in China and 'FBRI: EpiVacCorona' in Russia were approved. ${ }^{\text {s126,s127 }}$ Further, the mRNA vaccine 'Takeda: TAK-919' was recently approved in Japan, which contains the same formulation as Moderna's mRNA-1273 vaccine. ${ }^{16}$ A total of four inactivated vaccines were licensed in China ('Sinopharm [Beijing] BBIBPCorV', 'Sinopharm [Wuhan] Inactivated (Vero Cells)', 'Minhai Biotechnology: SARS-CoV2 Vaccine (Vero Cells)' and 'Sinovac CoronaVac'), and an inactivated vaccine in India ('Bharat Biotech Covaxin'), an inactivated vaccine in Russia (Chumakov Center KoviVac) and an inactivated vaccine in Kazakhstan (Kazakhstan RIBSP [Research Institute for Biological Safety Problems] QazCovid-in) have been approved by various national authorities. ${ }^{16}$ s128-s130

Table 1 provides a more detailed overview of the current phase III vaccine candidates and vaccines already approved.

BNT162b2 has already been approved by the EMA (21 December 2020), MHRA (30 December 2020), FDA (11 December 2020), HC (9 December 2020) and TGA (25 January 2021). Moreover, it is already being used in 85 countries, making it the most widely used COVID-19 vaccine worldwide. ${ }^{5131}$ The vaccine showed an efficacy of 95\% in preventing COVID-19. Side effects were mainly limited to local reactions and mild systemic reactions. Four related AEs were reported (shoulder injury related to vaccine administration, right axillary lymphadenopathy, paroxysmal ventricular arrhythmia and right leg paresthesiae). ${ }^{18}$

mRNA-1273 has been approved by the EMA (6 January 2021), MHRA (8 January 2021), FDA (18 December 2020) and HC (23 December 2020). The vaccine showed an efficacy of $94.1 \%$ in preventing COVID-19 and 100\% in preventing severe COVID19. The safety profile is comparable with that of BNT162b2, ${ }^{19}$ and risk groups, including immunocompromised patients with HIV infection, were also enrolled. Facial palsies occurred in $<0.1 \%$ of cases in the vaccination group (three cases) and the control group (one case). For BNT162b2 an additional first safety update was published on 28 January 2021, raising no new safety aspects. ${ }^{\text {s132 }}$

ChAdOx1 nCoV-19 has been approved by the EMA (29 January 2021), MHRA (30 December 2020), HC (26 February 2021) and TGA (16 February 2021). On 22 March 2021, 


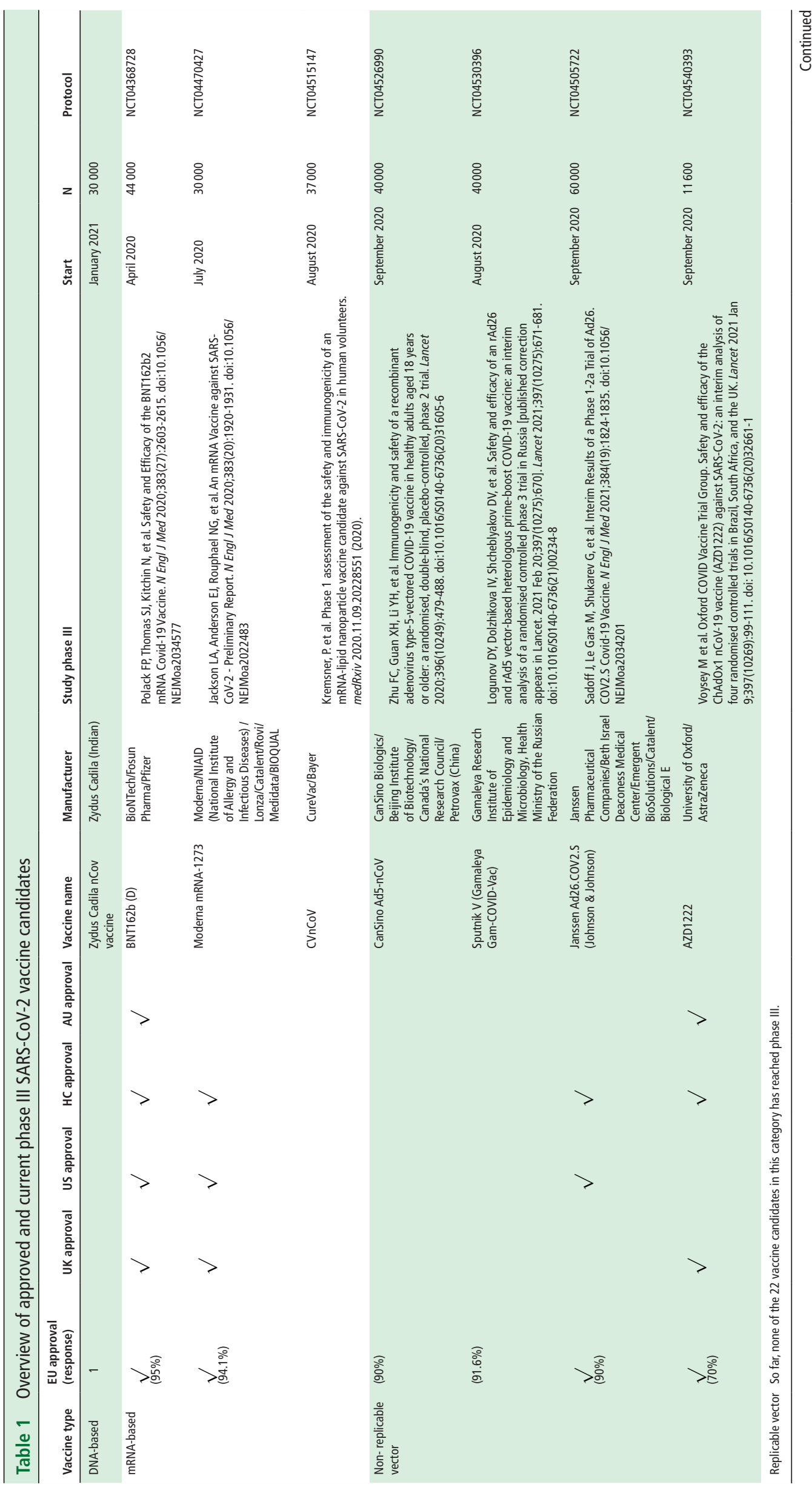




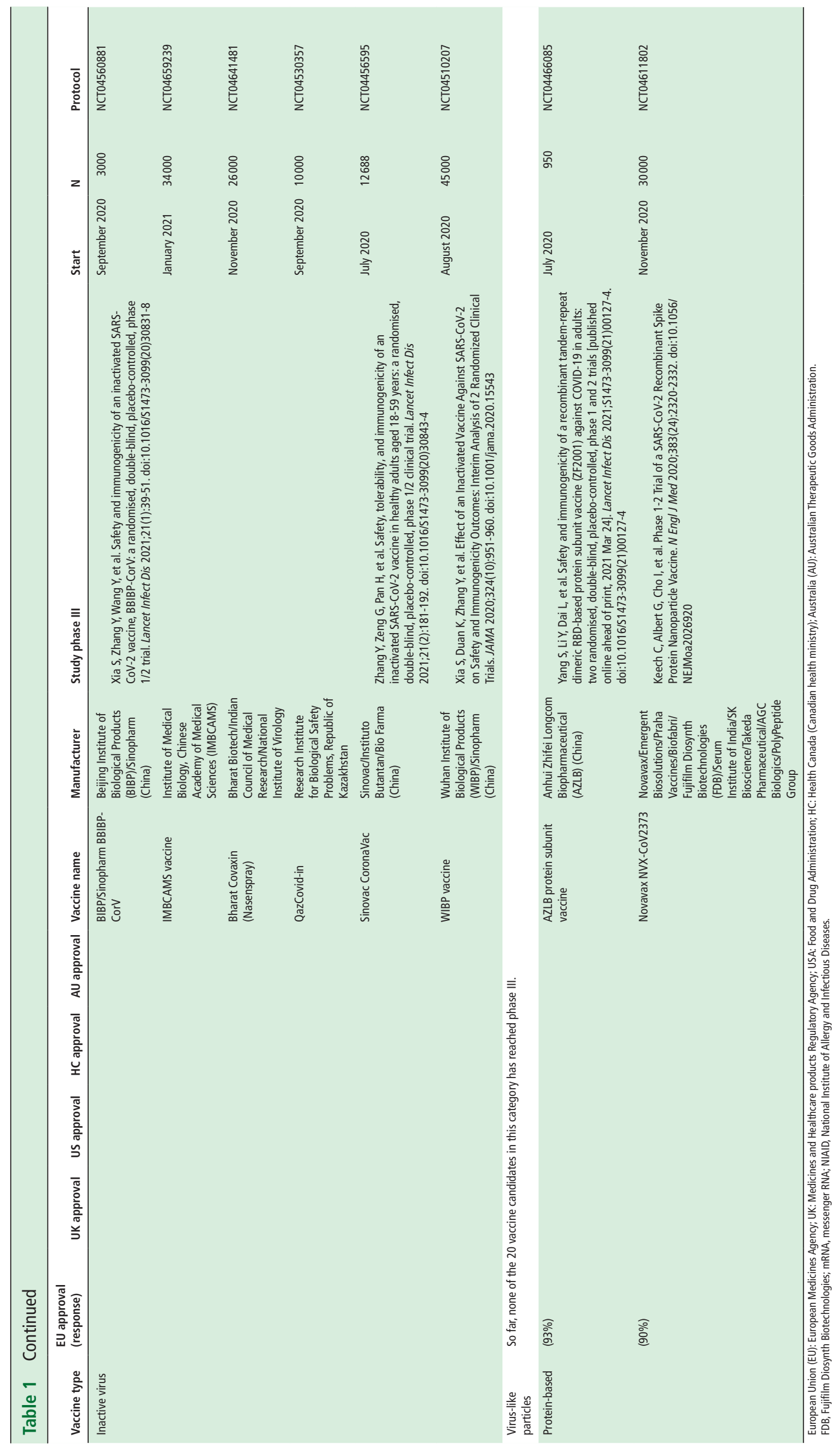


results from AstraZeneca's US phase III trial of AZD1222 were published, showing a statistically significant $79 \%$ efficacy in preventing symptomatic COVID-19 and 100\% efficacy in preventing severe disease and hospitalisation. ${ }^{.133,5134}$ The vaccine showed an efficacy of $70 \%$ against symptomatic disease. ${ }^{\text {s105 }}$ While this vector vaccine had a similarly reassuring safety profile, three cases of transverse myelitis were reported in the phase III clinical trial. ${ }^{.05,5135}$ This was assessed as unlikely to be related to the vaccination by an independent neurological expert committee. ${ }^{\text {s105 }}$ Approval by the EMA was granted on 29 January 2021. ${ }^{\mathrm{s} 136}$

As of 4 April 2021, a total of 169 cases of cerebral venous thrombosis (CVST) and 53 cases of splanchnic venous thrombosis have been reported to EudraVigilance. By this date, approximately 34 million people in the EEA (European Economic Area) and the UK had been vaccinated. The recent data do not change the recommendations of the EMA's safety committee (PRAC [Pharmacovigilance Risk Assessment Committee]) for the ChAdOx1 nCoV-19 (Vaxzevria) vaccine, emphasising the ascertainment that the overall benefit to risk ratio remains positive. ${ }^{s 137}$ Additionally, a safety update published by PRAC on 20 May 2021 recommends not revaccinating individuals with Vaxzevria who have developed thrombocytopaenia syndrome after their first vaccination with Vaxzevria. In addition, PRAC advises that individuals diagnosed with thrombocytopaenia within 3 weeks of vaccination should be actively screened for signs of thrombosis. Vice versa, individuals who develop thrombosis within 3 weeks of vaccination should be screened for thrombocytopaenia. ${ }^{\text {s138 }}$ In this context, a paper by Greinacher et al reported nine patients (eight women; median age 36, range, 22-49) with thrombosis starting 4-16 days after vaccination: seven patients had CVST, one had pulmonary embolism and one had splanchnic venous thrombosis and CVST; four patients died. The formation of platelet activating antibodies has been reported as a possible mechanism for postvaccination prothrombotic thrombocytopaenia in some cases. ${ }^{\text {s139-s145 }}$ In a nested incident-matched casecontrol study from Scotland, a first dose of ChAdOx1 nCoV-19 but not BNT162b2 was associated with a slightly elevated risk of developing idiopathic thrombocytopaenic purpura, haemorrhagic events and arterial thrombosis. ${ }^{\text {sit }}$

Ad26.COV2.S (Janssen/Johnson \& Johnson) has been recently approved by the EMA (11 March 2021), FDA (27 February 2021) and HC (5 March 2021). ${ }^{\text {s147 }}$ The vaccine showed 66\% efficacy in preventing the combined study endpoints of moderate and severe COVID-19, according to data published so far. ${ }^{\text {s148 }}$ Particularly in the prevention of severe/critical COVID-19, an efficacy of up to $90 \%$ could be shown. Furthermore, according to the approval data, there were no special safety aspects. ${ }^{\text {s121,s122 }}$ However, six cases of CVST have also been reported occurring after administration of the Janssen/Johnson \& Johnson vector vaccine. The benefits of vaccination outweigh these risks and therefore, according to a recent review by the FDA and the CDC (Centers for Disease Control and Prevention), has not prompted withdrawal of emergency approval. .140,s149-s151 $^{\text {s. }}$

Moreover, an initial study by Shimabukuro et al found no safety issues related to mRNA vaccines in pregnant women, although more data are needed. ${ }^{\text {s152 }}$

In addition, recent studies provide evidence for a protective immunity lasting 9 months after SARS-CoV-2 infection, although more extensive investigations are needed. ${ }^{\text {s153-s156 }}$

Potential long-term side effects of the new genetic vaccines (nucleic acid and vector-based vaccines) cannot be assessed at present and require appropriate alertness in everyday clinical practice. ${ }^{\text {s157-s160 }}$ Although, at least for RNA vaccines, the RNA is likely to remain fairly local (eg, in the muscles) and will be degraded fairly quickly, distal effects/complications seem very unlikely.

The emergence of viral mutants escaping immune control is another important issue in the context of the COVID-19 pandemic. Variants of concern are those that have an impact on diagnostics, therapy and vaccination and are associated with increased transmissibility and greater disease severity. These currently include variants B.1.1.7 (first detected in England), B.1.351 (first detected in South Africa), P.1 (first detected in Brazil), and B.1.427 and B.1.429 (first detected in the USA/ California). ${ }^{\text {s161-s163 }}$ Another problem variant is the mutant strain B.1.617 (first detected in India). ${ }^{\text {s164 }}$ Consequences may encompass reduced vaccine efficacy, especially against the B.1.351 strain, and breakthrough infections in vaccinated persons as well

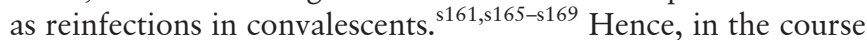
of fighting the pandemic, it is of utmost importance to win the race between viral evolution and global vaccination coverage to guarantee long-term immune control of the SARS-CoV-2 pandemic. Annual adaptation of the vaccines to the actual circulating virus variants, similar to what has become routine practice with influenza, could be an appropriate solution. ${ }^{\text {.167 }}$

In the age of vaccination scepticism, it is once again of paramount importance for neurologists to emphasise to their patients that numerous epidemiological studies have failed to provide evidence for causation or triggering onset of MS by vaccination. Additionally, there is no evidence that vaccination induces MS attacks, with the exception of case series linking yellow fever live vaccine with relapse. ${ }^{5170}$ The typhus vaccine may even have a positive influence on MS disease course. ${ }^{5171}$ In line with this, a study by Hapfelmeier $e t a^{1572}$ suggested that vaccination is associated with a lower probability of being diagnosed with MS within the next 5 years.

As these infections clearly carry a relapse/pseudo-relapse risk, not to mention potentially very serious outcomes, including death in the case of SARS-CoV-2 and COVID-19, it is therefore essential to vaccinate and thus protect patients with MS. ${ }^{.171,5173,5174}$

This should always be based on a comprehensive evaluation of risks and benefits and consideration of the person's immune status, including ruling out potential contraindications such as the use of attenuated vaccines in patients on immunosuppressive therapy.

\section{DMTS AND VACCINES}

Immunisation status should be assessed in patients before starting immunosuppressive regimens. ${ }^{\text {s175, } 176}$ It is worth noting that the absence of a pathogen-specific antibody response does not necessarily indicate lack of protection given the role of $\mathrm{T}$ lymphocyte responses combating viral infections. In principle, inactivated vaccines should be administered at least 2 weeks and live vaccines 4 weeks (ideally 6 weeks) before initiating immunotherapy. ${ }^{\text {s177,s178 }}$ In MS, vaccinations should be undertaken according to local vaccine recommendations. ${ }^{5178,5179}$ In the case of the yellow fever vaccination, which is obligatory for travelling to certain countries, a risk-benefit assessment must be carried out, as relapse may be potentially triggered by vaccination..$^{\text {s170,s171 }}$

For certain DMTs, for example, B cell-depleting therapies, a conflicting situation may arise with balancing the need to contain MS activity and the need to convey protection through vaccination. It may not be practical to wait 6 months to vaccinate in the case of ocrelizumab, for example. Therefore, clinical practice-oriented recommendations are necessary. Table 2 is intended to provide an overview of the recommendations for 
Table 2 DMT and vaccination recommendations for live/non-live/gene-based vaccines as well as the recommended time of administration and expected immune response

\begin{tabular}{|c|c|c|c|c|c|c|c|c|}
\hline \multirow[b]{2}{*}{ DMT } & \multirow{2}{*}{$\begin{array}{l}\text { Live } \\
\text { vaccines }^{178,5180 \text {-s } 183}\end{array}$} & \multirow{2}{*}{$\begin{array}{l}\text { Non-live } \\
\text { vaccine }\end{array}$} & \multicolumn{2}{|c|}{$\begin{array}{l}\text { Gene-based } \\
\text { vaccine }{ }^{s 89, s 182}\end{array}$} & \multirow{2}{*}{$\begin{array}{l}\text { Timing of vaccine after } \\
\text { DMT is stopped* }\end{array}$} & \multirow{2}{*}{$\begin{array}{l}\text { Timing of vaccine } \\
\text { after DMTt }\end{array}$} & \multirow{2}{*}{$\begin{array}{l}\text { Timing of DMT } \\
\text { after vaccine } \ddagger^{s 183}\end{array}$} & \multirow[b]{2}{*}{ Immune response§ } \\
\hline & & & mRNA & Vector & & & & \\
\hline HDMP & Contraindicated & Yes & Yes & Yesף & $\geq 1$ month $^{\mathrm{s} 184}$ & Therapy stopped* * & $\geq 2-4$ weeks $\ddagger$ & May be reduced ${ }^{5185}$ \\
\hline Interferon & Strict indication & Yes & Yes & Yesף & Anytime ${ }^{\text {s186 }}$ & Anytime & 22-4 weeks & Similar ${ }^{5187}$ \\
\hline Glatiramer acetate & Strict indication & Yes & Yes & Yesף & Anytime $^{\mathrm{s} 186}$ & Anytime & $\geq 2-4$ weeks & Similar ${ }^{5188}$ \\
\hline Dimethyl fumarate & Strict indication & Yes & Yes & Yesף & Not specified & Anytime & $\geq 2-4$ weeks $\ddagger$ & Similar ${ }^{5189}$ \\
\hline Teriflunomide & Contraindicated & Yes & Yes & Yesף & $\geq 6$ months ${ }^{\mathrm{s} 190}+\dagger$ & Anytime & $\geq 2-4$ weeks & Slightly reduced ${ }^{\mathrm{s} 191, \mathrm{~s} 192}$ \\
\hline S1P modulators $\ddagger \ddagger$ & Contraindicated & Yes & Yes & Yesף & $\geq 2$ months $^{\mathrm{s} 193}$ & Anytime & $\geq 2-4$ weeks $\ddagger$ & Reduced $^{\text {s194-s196 }}$ \\
\hline Natalizumab & Contraindicated & Yes & Yes & Yesף & $\geq 3$ months $^{5186}$ & Anytime & $\geq 2-4$ weeks $\ddagger$ & Similar ${ }^{5197,5198}$ \\
\hline $\begin{array}{l}\text { B cell-depleting } \\
\text { agents } \S \S\end{array}$ & Contraindicated & Yes & Yes & Yesף & Specifiedๆף & $\geq 3-6$ months ${ }^{* *}$ & $\geq 2-4$ weeks $\ddagger$ & Reduced $^{\mathrm{s} 196, \mathrm{~s} 199, \mathrm{~s} 200}$ \\
\hline Alemtuzumab & Contraindicated & Yes & Yes & Yesף & Not specifieds178,s201 & $\geq 3-6$ months*** & $\geq 2-4$ weeks $\ddagger$ & Reduced $^{\mathrm{s} 202}$ \\
\hline Cladribine & Contraindicated & Yes & Yes & Yesף & Specifiedt†† & 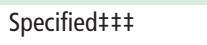 & $\geq 2-4$ weeks $\ddagger$ & Similar ${ }^{5196, s 203}$ \\
\hline Mitoxantrone & Contraindicated & Yes & Yes & Yesף & $\geq 3$ months ${ }^{\text {s204 }}$ & Not specified & $\geq 2-4$ weeks $\ddagger$ & May be reduced ${ }^{\text {s178,s204 }}$ \\
\hline
\end{tabular}

${ }^{*}$ Recommended timing of vaccination for live/attenuated vaccines after stopping DMT, with timing meant to avoid the risk of infection from the vaccine itself in immunocompromised individuals.

†Recommended timing of vaccination for non-live/gene-based vaccines with already established DMT (concerning cyclical therapies after last administration of DMT) to generate the most protective vaccine response possible.

¥Recommended timing of the start of a DMT/next dose of a DMT (especially concerning cyclical therapies) after completion of the vaccine to enable a protective vaccine response before the immune response is possibly affected by the DMT/next dose of DMT-at least 2 weeks for non-live/gene-based vaccines and at least 4 weeks for live/ attenuated vaccines (here also for safety reasons).

$\S$ Data only for non-live vaccines available.

III Non-replicating viral vector vaccines.

**III After end of therapy or after dose reduction of prednisone equivalent $<20 \mathrm{mg} /$ day in adults. ${ }^{\text {s184 }}$

t†IV Washout option with active carbon or cholestyramine, regardless of the washout procedure chosen, a subsequent check of the plasma level by two separate tests at least 14 days apart, and a waiting period of 1.5 months between the first measurement of a plasma level below $0.02 \mathrm{mg} / \mathrm{L}$ and the live vaccination is required. ${ }^{\text {s } 190}$

$\ddagger \neq a$ Sphingosine-1-phosphate (S1P) receptor modulators including fingolimod, siponimod, ozanimod and ponesimod.

$\S \S b$ Including rituximab, ocrelizumab and ofatumumab.

IIV At least 6 months for revaccination and 12 months for primary vaccination, if possible ${ }^{5184}$ respectively until B cell recovery. ${ }^{5178}$

$* * *$ VI At least 3-6 months apart depending on regional recommendations. ${ }^{\text {s94,s205-s207 }}$

†††VII Until white cell counts are within normal limits. ${ }^{\text {s208 }}$

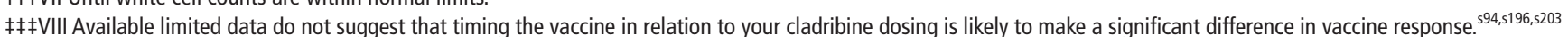

DMT, disease-modifying therapy; HDMP, high-dose methylprednisolone; mRNA, messenger RNA.

live, inactivated and currently licensed gene-based COVID-19 vaccines, with particular reference to DMT.

A major limitation of all known vaccination clinical trials in the context of the SARS-CoV-2 pandemic is that patients with autoimmune diseases on immunotherapy have not been specifically considered. Therefore, obviously, our evidence-based knowledge is currently limited.

\section{Glucocorticoids}

For live vaccines, no therapy stop is required under glucocorticosteroid treatment if (1) local therapy or (2) short-term (<14 days) and low-dose therapy (prednisone equivalent $<20 \mathrm{mg}$ /day in adults) is used. For therapy durations, longer than 14 days and above the prednisone equivalent mentioned, an interval between last intake and vaccine administration of 1 month is recommended for live vaccines to avoid an increased vaccination-related reaction. Inactivated vaccines do not bear an increased risk at all. However, they should only be administered after therapy has been stopped or the dose reduced to the prednisone equivalent mentioned due to the possible reduced efficacy. ${ }^{\text {s184 }}$ No data regarding patients with MS are available to date. In conclusion, no increased risk is expected with mRNA and non-replicating viral vector vaccines.

\section{Interferons}

With reference to regional recommendations, live vaccines are not contraindicated during therapy with interferon- $\beta$ preparations, but a risk-benefit assessment should be carried out. ${ }^{\text {s180,s181,s186 }}$ Regarding inactivated vaccines, there is no increased risk in view of the mode of action. Concerning vaccine efficacy, the immune response to an influenza vaccine in patients with MS did not differ from controls. ${ }^{\text {s187 }}$ Overall, based on the comparability of inactivated vaccines and gene-based vaccines, no heightened risk is expected with mRNA as well as non-replicating viral vector vaccines and a comparable immune response can be assumed.

\section{Glatiramer acetate}

There is no contraindication for live vaccines during glatiramer acetate treatment, but a risk-benefit assessment is required. ${ }^{\mathrm{s} 186}$ Concerning inactivated vaccines, a similar immune response after vaccination compared with controls could be shown. ${ }^{\text {s188 }}$ Overall, no increased risk is expected with mRNA and non-replicating viral vector vaccines as it is also the case for inactivated vaccines.

\section{Dimethyl fumarate}

Based on regional recommendations, there is no absolute contraindication for live vaccines during therapy with dimethyl fumarate (DMF). Nevertheless, a strict risk-benefit calculation is advised. ${ }^{\text {s180,s181 }}$ For inactivated vaccines, in a study comparing $D M F$ with non-pegylated interferon- $\beta$ regarding a recall antigen ( $\mathrm{T}$ cell-dependent), a $\mathrm{T}$ cell independent antigen (PPSV23) and a neoantigen (MCV4), an overall comparable immune response was observed. However, T cell response was diminished in relation to the $\mathrm{T}$ cell-dependent vaccine in comparison 
with interferon- $\beta .{ }^{s 189}$ Overall, given the mechanism of action of both inactivated and gene-based vaccines, no increased risk is therefore expected with mRNA and non-replicating viral vector vaccines.

\section{Teriflunomide}

Live vaccines are contraindicated during treatment with teriflunomide and according to the prescribing information for at least 6 months after the end of therapy. ${ }^{s 190}$ However, if necessary, a washout with active carbon $(50 \mathrm{~g}$ four times daily for 11 days) or cholestyramine (8g three times daily for 11 days) can be performed. Regardless of the washout procedure, a subsequent check of the plasma level by two separate tests at least 14 days apart and a latency of 1.5 months between the first measurement of a plasma level below $0.02 \mathrm{mg} / \mathrm{L}$ and injection of the live vaccine is required. ${ }^{\text {s184 }}$ For inactivated vaccines, there are generally no safety problems regarding DMTs. In a study by Bar-Or et al, vaccination with a neoantigen (rabies vaccine) showed sufficient immunity in the teriflunomide (under treatment) and control groups. ${ }^{\text {s192 }}$ However, the immune response was significantly reduced in the teriflunomide group. This confirms the results from an influenza vaccination study where the immune response was compared between teriflunomide and interferon- $\beta$. Similarly, both groups achieved disease-protective immunity (titres $\geq 40$ ), but the immune response to the H1N1 and H3N2 strains was significantly weaker in teriflunomide-treated patients. ${ }^{\text {s191 }}$ Overall, no elevated risk is expected with mRNA and non-replicating viral vector vaccines, although reduced immune response may occur.

\section{S1P-R modulators (fingolimod, siponimod, ozanimod, ponesimod)}

Live vaccines are contraindicated in patients receiving S1P-R (sphingosine-1-phosphate-receptor) modulators. DMT should be stopped at least 2 months before a live vaccine can safely be administered due to the prescribing information. ${ }^{\text {s193 }}$ Administration of inactivated vaccines, as with all DMTs, does not carry an increased risk, but leads to significantly impeded immune responses. ${ }^{\text {s180,s181 }}$ The immune response to the inactivated influenza vaccine $(54 \%$ vs $85 \%$ at 3 weeks and $43 \%$ vs $75 \%$ at 6 weeks postvaccination), as well as the tetanus toxoid (TT) vaccine $(40 \%$ vs $61 \%$ at 3 weeks and $38 \%$ vs $49 \%$ at 6 weeks postvaccination), was significantly reduced in individuals on fingolimod treatment. ${ }^{\text {s194 }}$ Similar results were shown for siponimod. ${ }^{\text {s209 }}$ No data are yet available for ozanimod and ponesimod, but comparability can be assumed. In a preliminary study from Israel, only $3.8 \%$ of 26 patients with MS on fingolimod treatment developed a protective humoral immune response to BNT162b2. ${ }^{\mathrm{s} 196}$ This observation is compatible with the mode of action of sphingosine-1-phosphate receptor modulators since on receptor engagement $\mathrm{T}$ helper cells delivering signals to $\mathrm{B}$ cells to manufacture antibodies are restrained in the lymph nodes. ${ }^{\text {s210 }}$ Overall, based on the mode of action, no elevated risk is expected with mRNA and non-replicating viral vector vaccines, although reduced immunogenicity may occur.

\section{Natalizumab}

Live vaccines are contraindicated during immunomodulatory therapy with natalizumab. Therefore, a minimum interval of 3 months from end of therapy to administration of vaccination is recommended. ${ }^{\text {s186 }}$ Inactivated vaccines can be administered at any time during treatment with natalizumab. Furthermore, the immune response to an inactivated influenza vaccine as well as to the recall antigen (TT) and the neoantigen (keyhole limpet haemocyanin $(\mathrm{KLH})$ ) was investigated in two studies. All three showed similar immune responses compared with the control

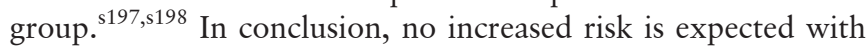
mRNA as well as non-replicating viral vector vaccines and a similar immune response is likely.

\section{B cell-depleting therapies (ocrelizumab, rituximab,} ofatumumab)

Live vaccines are contraindicated in patients treated with B cell-depleting therapies. Here, a minimum interval of 6 months for booster vaccination and 12 months for initial vaccination should be observed, if possible respectively postponed until B cell recovery. ${ }^{\text {s178,s184 }}$

Regarding inactivated vaccines, the immune response to the TT vaccine, 23-valent pneumococcal polysaccharide vaccine (23PPV), influenza vaccine and KLH vaccine was investigated with ocrelizumab in the VELOCE study (the control group included patients without therapy or with interferon- $ß$ ). The responder rate for the TT vaccine after 8 weeks was $23.9 \%$ vs $54.5 \%$ (ocrelizumab vs control), for the 23-PPV vaccine after 4 weeks $71.6 \%$ vs $100 \%$ (ocrelizumab vs control), and for the influenza vaccine (five strains) after 4 weeks was $55.6 \%$ (80\% in the ocrelizumab group and 75\%-97\% in the control group). For the KLH vaccine, the humoral immune response was also significantly weakened. ${ }^{\text {s199 }}$ In addition, the immune response to TT, pneumococcal polysaccharide and KLH vaccines was investigated in a study with patients with rheumatoid arthritis under rituximab and methotrexate versus only methotrexate. Immune responses to the $\mathrm{T}$ cell-dependent protein antigen TT were similar under rituximab after 24 weeks. For the neoantigen $(\mathrm{KLH})$ and the $\mathrm{T}$ cell-independent pneumococcal vaccine antigen, the immune responses were significantly reduced.

Initial studies in MS and other conditions suggest a diminished antibody response to SARS-CoV-2 under B cell-depleting therapies..$^{\text {2211-s } 215}$ In a study by Achiron et al, only $22.7 \%$ of 44 patients treated with ocrelizumab developed a protective humoral immune response to BNT162b2. ${ }^{\text {s196 }}$ It should be emphasised that absence of humoral immunity does not necessarily imply absence of any protection given the important role of CD4 and CD8 T lymphocytes in combating viral infections including COVID-19. ${ }^{\text {s216-s2 } 218}$

Following this and according to regional recommendations, it is reasonable when considering to start or continue B celldepleting therapies to coordinate the timing of the vaccination with the next treatment to ensure generation of a protective immune response. For this purpose, an interval of at least 3-6 months after the last administration of DMT is recommended. ${ }^{\mathrm{s} 205, \mathrm{~s} 207}$ For ofatumumab, no data are available to date. In general, no increased risk is expected with mRNA and nonreplicating viral vector vaccines as is the case for inactivated vaccines, although a dampened immune response can be anticipated (excluding $\mathrm{T}$ cell-dependent immune responses).

When assessing the individual potential risk-benefit equation, one should take into account disease activity, age and comorbidities. In an older patient with additional comorbidities, the imperative to protect from COVID-19 may be particularly high, and the 'MS disease-activity/progression-risk' may be minimal over a few months. Hence, even delaying the next infusion can be sensible in order to maximise vaccine-induced protection. ${ }^{92}$ In younger persons with no comorbidities but very active MS, maintaining optimal MS control may be viewed as more important than immediate protection from COVID-19. 


\section{Alemtuzumab}

Live vaccines are contraindicated during and after 'recent' therapy with alemtuzumab, with no specific defined minimal interval. ${ }^{\text {s178,s201 }}$ For inactivated vaccines, in a study by McCarthy et al,pretherapeutically acquired immunities were maintained under immunosuppression with alemtuzumab. ${ }^{\text {s202 }}$ On the other hand, vaccinations within 6 months after start of therapy led to reduced immunity. According to the recommendations of the MSIF, an inactivated vaccine should be carried out at the earliest 3 months after the last administration. ${ }^{899}$ Accordingly, no increased risk is expected with mRNA and non-replicating viral vector vaccines, although a reduced immunity within 6 months after last administration can occur.

\section{Cladribine}

Live vaccines are generally contraindicated during immunotherapy with cladribine. According to the EMA, a live vaccine can be administered as soon as the leucocytes are within the normal range. ${ }^{\text {s208 }}$ Following local recommendations, an inactivated vaccine should be administered at the earliest 3-6 months after the last administration to keep the probability of an adequate immune response as high as possible. ${ }^{\text {s89,s205,s207 }}$ To date, only preliminary data on vaccination responses during or after cladribine therapy have been published.

Nevertheless, an analysis from the MAGNIFY-MS study presented at ACTRIMS (Americas Committee for Treatment and Research in Multiple Sclerosis) 2021 showed that protective antibody titres do develop after influenza and varicella-zoster vaccination under therapy with cladribine. ${ }^{\mathrm{s} 203}$ The recent report from Israel by Achiron et al demonstrated that there was no reduced humoral immune response to BNT162b2 in patients undergoing cladribine treatment. ${ }^{196}$ Further studies on larger cohorts are needed to confirm this observation. In conclusion, no increased risk is expected with mRNA and non-replicating viral vector vaccines as is the case with inactivated vaccines.

\section{Mitoxantrone}

Live vaccines are contraindicated during immunotherapy with mitoxantrone. A minimum interval of 3 months after the end of therapy is recommended. ${ }^{\text {s204 }}$ This also seems reasonable for inactivated vaccines in order to be able to develop sufficient immunity. In a study by Olberg et al, $0 \%$ of patients with MS on mitoxantrone therapy gained immunity to an inactivated influenza vaccine. ${ }^{\text {s219 }}$ In summary, no increased risk is expected with mRNA and non-replicating viral vector vaccines as it is with inactivated vaccines, although vaccine immunogenicity may be compromised.

\section{RECOMMENDATIONS FOR VACCINATION IN MS IN THE COVID-19 ERA}

In the recent past, 17 SARS-CoV-2 vaccines have been approved worldwide. ${ }^{16}$ The two mRNA vaccines (BNT162b2, mRNA1273) and the vector vaccine ChAdOx $1 \mathrm{nCoV}-19$ represent the most widely used COVID-19 vaccines worldwide. ${ }^{\text {s131 }}$ For these three vaccines, excellent efficacy was shown in approval studies, although the efficacy of the ChAdOx $1 \mathrm{nCoV}-19$ vaccine was $70 \%$, worse than that of the two mRNA vaccines $(95 \%$ for BNT162b2, 94.1\% for mRNA-1273). ${ }^{\text {s22 }}$ Additionally, the two mRNA vaccines had a particularly good safety profile. A safety update on the BNT162b2 vaccine was published on 28 January 2021 which did not raise any new safety issues. ${ }^{\text {s132 }}$ In addition, BNT162b2 was emergency-approved by the FDA on 10 May 2021 and by the EMA on 28 May 2021 for adolescents aged $12-15$ years. ${ }^{\text {s221-s223 }}$ Regarding the vector vaccine ChAdOx1 $\mathrm{nCoV}-19$, three cases of transverse myelitis were reported in the verum group, although these were considered unlikely to be related to vaccination by an independent expert committee. ${ }^{\text {s105 }}$ According to a pivotal study, one case of myelitis most likely corresponds to previously undiagnosed MS, one is related to meningococcal vaccination and one case requires further clarification. ${ }^{\text {s105 }}$ It should also be emphasised that even though only $9.8 \%$ of the patients included in the pivotal study were aged over 65 years, there is still a clear WHO recommendation for its use in this age group. ${ }^{\text {.105,s224 }}$ This can be justified by both the excellent safety profile in the pivotal study and by evidence of generally comparable immunity in the elderly. ${ }^{\text {s22 }}$ Additionally, real-world data from Scotland show a 94\% reduction in hospitalisations (majority over 80 years old) following vaccination with ChAdOx1 nCoV-19. ${ }^{\text {s2 } 26}$ Another real-world data analysis from Scotland showed an 88\%-91\% reduction in COVID19-related hospitalisations for the BNT162b2 and ChAdOx1 $\mathrm{nCoV}-19$ vaccines as early as $28-34$ days after the first vaccination. ${ }^{\text {s27 }}$ This is confirmed by an analysis among healthcare workers in England. ${ }^{\text {s228 }}$

The second promising vector vaccine Gam-COVIDVac $=$ Sputnik V was approved in August 2020 in Russia based on a phase II trial. On 2 February 2021, phase III trial data were published showing 91.6\% efficacy in preventing COVID-19. Safety data were also comparable with the mRNA vaccines. ${ }^{s 123}$ Furthermore Ad26.COV2.S as a vector vaccine has been approved recently by the FDA and EMA. ${ }^{\text {s121,s122 }}$ According to preliminary data from Janssen/Johnson \& Johnson, with up to 90\% efficacy in preventing severe/critical COVID-19 the vaccine shows high efficacy. ${ }^{\text {s148 }}$ Moreover there were no major safety signals documented in the approval data. ${ }^{\text {s121,s122 }}$

It is important to recognise that Gam-COVID-Vac=Sputnik V, ChAdOx1 nCoV-19 and Ad26.COV2.S are all non-replicating vector vaccines, the first being a combination of two different recombinant human adenoviruses, the second being a chimpanzee virus and the third being a human adenovirus type 26 (Ad26), and therefore cannot cause a vector virus infection.

Several vaccines have been associated with secondary immune disorders, such as GBS in the case of the 1976 H1N1 vaccine or immune thrombocytopaenia in the case of the measles-mumpsrubella vaccine. ${ }^{\text {s229,s230 }}$ The Vaccine Adverse Event Reporting System database yielded a GBS rate of 0.7 per 1 million for influenza vaccines and showed that among all vaccines GBS occurs most frequently with influenza vaccines. ${ }^{\text {s231,s232 }}$ However, it is not yet clarified whether there is a causal relationship.

In the context of SARS-CoV-2 vaccines, as discussed earlier, a presumably antibody-mediated prothrombotic thrombocytopaenia with cerebral venous and splanchnic venous thrombosis was observed in a small fraction of vaccinees. ${ }^{\text {s139 }}$ Therefore, an appropriate surveillance programme is mandatory to detect serious and potentially lethal AEs like these.

Nevertheless, the safety of gene-based vaccines has been evaluated in the past as well as in recent phase III trials, and monitoring did not raise other major safety concerns (except as discussed in the three cases of myelitis in the ChAdOx1 nCoV-19 study). ${ }^{\text {s105,s123,s233-s236 }}$

Based on current recommendations of the MSIF, SARS-CoV-2 vaccination (with $\mathrm{mRNA}$ and non-replicating viral vector vaccines) is advised for all patients with MS. ${ }^{889}$, s94,s174,s234,s237,s238 The timing of vaccination is generally the same as for inactivated vaccines (see table 2). For cyclical therapies (rituximab, ocrelizumab, alemtuzumab, cladribine), a minimum interval of 3 months after the last administration is reasonable to facilitate a sufficient immunological response. ${ }^{\mathrm{s} 239}$ In line with that, the next dose of cyclical 
therapies should be infused no earlier than 2-4 weeks after vaccination. Neurologists should be cognisant of a likely reduction in the immune response for non-T cell-dependent antigens in patients treated with some DMTs, in particular the B cell-depleting therapies. ${ }^{\text {s199 }}$

\section{CONCLUSION}

To efficiently fight the COVID-19 pandemic, it is of utmost importance to follow the general recommendations of the WHO and to achieve the fastest possible protection through comprehensive vaccination of the human population. ${ }^{5}$ s240 This is particularly relevant to people with autoimmune diseases such as MS. Therefore, all people with MS should be vaccinated against SARS-CoV-2 according to regional recommendations and in accordance with their treating neurologist, not least in order to prevent the emerging problem of virus mutants as far as possible. ${ }^{.89,594, s 205,5207,5241}$ Regarding cyclical therapies (ocrelizumab, rituximab, alemtuzumab, cladribine), possible postponement of the next dose may be considered depending on individual circumstances (risk factors, age, MS disease activity and prognosis, and lymphocyte count). ${ }^{589,592}$ The risk of disease reactivation may greatly exceed the risk of COVID $-19 . .^{\text {s242 }}$

Please see online supplemental file 1 for references 41-242.

\author{
Author affiliations \\ ${ }^{1}$ Department of Neurology, Medical University of Vienna, Vienna, Austria \\ ${ }^{2}$ Department of Neurology, Universitätsklinikum Düsseldorf, Medical Faculty, Heinrich \\ Heine University, Düsseldorf, Germany \\ ${ }^{3}$ Brain and Mind Centre, The University of Sydney, Sydney, New South Wales, \\ Australia \\ ${ }^{4}$ Department of Neurology, Neuroimmunological Section, University of Rostock, \\ Rostock, Germany \\ ${ }^{5}$ Department of Neuroinflammation, Queen Square Multiple Sclerosis Centre, \\ University College London (UCL) Queen Square Institute of Neurology, Faculty \\ of Brain Sciences, UCL; National Institute for Health Research, University College \\ London Hospitals, Biomedical Research Centre, London, UK \\ ${ }^{6}$ Department of Neurology, Center for Neuroinflammation and Experimental \\ Therapeutics, and Department of Neurology, Perelman School of Medicine, University \\ of Pennsylvania, Philadelphia, PA, USA
}

Contributors Conception and design of the work: TM, H-PH, PSR and UZ. Data analysis and interpretation: TM, H-PH, TZ, MB, NB, TB, JC, AB-O, PSR and UZ. Drafting the article: TM and $\mathrm{H}-\mathrm{PH}$. Critical revision of the article: TM, H-PH, TZ, MB, NB, TB, JC, $A B-0, P S R$ and UZ. Final approval of the version to be published TM, $H-P H, T Z, M B$, $N B, T B, J C, A B-O, P S R$ and UZ.

Funding The authors have not declared a specific grant for this research from any funding agency in the public, commercial or not-for-profit sectors.

Competing interests None declared.

Patient consent for publication Not required.

Ethics approval As this is a review article, it does not involve studies on humans or animals and is therefore in accordance with national law and the 1975 Declaration of Helsinki (as revised).

Provenance and peer review Not commissioned; externally peer reviewed. Supplemental material This content has been supplied by the author(s). It has not been vetted by BMJ Publishing Group Limited (BMJ) and may not have been peer-reviewed. Any opinions or recommendations discussed are solely those of the author(s) and are not endorsed by BMJ. BMJ disclaims all liability and responsibility arising from any reliance placed on the content. Where the content includes any translated material, BMJ does not warrant the accuracy and reliability of the translations (including but not limited to local regulations, clinical guidelines, terminology, drug names and drug dosages), and is not responsible for any error and/or omissions arising from translation and adaptation or otherwise.

Open access This is an open access article distributed in accordance with the Creative Commons Attribution Non Commercial (CC BY-NC 4.0) license, which permits others to distribute, remix, adapt, build upon this work non-commercially, and license their derivative works on different terms, provided the original work is properly cited, appropriate credit is given, any changes made indicated, and the use is non-commercial. See: http://creativecommons.org/licenses/by-nc/4.0/.

\section{ORCID iDs}

Tobias Monschein http://orcid.org/0000-0002-6053-4210

Tobias Zrzavy http://orcid.org/0000-0001-8909-1591

Michael Barnett http://orcid.org/0000-0002-2156-8864

Paulus Stefan Rommer http://orcid.org/0000-0001-5209-6647

\section{REFERENCES}

1 Monto AS, Fukuda K. Lessons from influenza pandemics of the last 100 years. Clin Infect Dis 2020;70:951-7.

2 Hamre D, Procknow JJ. A new virus isolated from the human respiratory tract. Exp Biol Med 1966;121:190-3.

3 Corman VM, Muth D, Niemeyer D. Hosts and sources of endemic human coronaviruses. In: Advances in Virus Research, 2018: 163-88.

4 JT W, Leung K, Leung GM. Nowcasting and forecasting the potential domestic and international spread of the 2019-nCoV outbreak originating in Wuhan, China: a modelling study. Lancet 2020;395:689-97.

5 WHO. WHO coronavirus (COVID-19) Dashboard, 2021. Available: https://covid19. who. int/.

6 Winkelmann A, Loebermann M, Reisinger EC, et al. Disease-Modifying therapies and infectious risks in multiple sclerosis. Nat Rev Neurol 2016;12:217-33.

7 Magyari M, Sorensen PS. Comorbidity in multiple sclerosis. Front Neurol 2020;11:851.

8 Hauer L, Perneczky J, Sellner J. A global view of comorbidity in multiple sclerosis: a systematic review with a focus on regional differences, methodology, and clinical implications. J Neurol 2020;19. doi:10.1007/s00415-020-10107-y. [Epub ahead of print: 27 Jul 2020].

9 Rostami Mansoor S, Ghasemi-Kasman M. Impact of disease-modifying drugs on the severity of COVID-19 infection in multiple sclerosis patients. J Med Virol 2021:93:1314-9.

10 Marrie RA, Elliott L, Marriott J, et al. Effect of comorbidity on mortality in multiple sclerosis. Neurology 2015;85:240-7.

11 Wouk J, Rechenchoski DZ, Rodrigues $B C D$, et al. Viral infections and their relationship to neurological disorders. Arch Virol 2021;166:733-53.

12 Möhn N, Konen FF, Pul R, et al. Experience in multiple sclerosis patients with COVID-19 and disease-modifying therapies: a review of 873 published cases. J Clin Med 2020;9:4067.

13 Pawlitzki M, Zettl UK, Ruck T, et al. Merits and culprits of immunotherapies for neurological diseases in times of COVID-19. EBioMedicine 2020;56:102822.

14 Sellner J, Rommer PS. Multiple sclerosis and SARS-CoV-2 vaccination: considerations for immune-depleting therapies. Vaccines 2021;9:99-12.

15 Korsukewitz C, Reddel SW, Bar-Or A, et al. Neurological immunotherapy in the era of COVID-19 - looking for consensus in the literature. Nat Rev Neurol 2020;16:493-505.

16 COVID-19 vaccine tracker. Available: https://covid19.trackvaccines.org/vaccines/.

17 . Available: https://www.canada.ca/en/health-canada/services/drugs-health-products/ covid19-industry/drugs-vaccines-treatments/vaccines.html

18 Polack FP, Thomas SJ, Kitchin N, et al. Safety and efficacy of the BNT162b2 mRNA Covid-19 vaccine. N Engl J Med 2020;383:2603-15.

19 Baden LR, El Sahly HM, Essink B, et al. Efficacy and safety of the mRNA-1273 SARSCoV-2 vaccine. N Engl J Med Overseas Ed 2021;384:403-16.

20 MacDonald NE, SAGE Working Group on Vaccine Hesitancy. Vaccine hesitancy: definition, scope and determinants. Vaccine 2015;33:4161-4.

21 Portaccio E, Fonderico M, Hemmer B, et al. Impact of COVID-19 on multiple sclerosis care and management: results from the European Committee for treatment and research in multiple sclerosis survey. Mult Scler 2021:135245852110053.

22 Lu R, Zhao X, Li J, et al. Genomic characterisation and epidemiology of 2019 novel coronavirus: implications for virus origins and receptor binding. The Lancet 2020;395:565-74.

23 Gorbalenya AE, Baker SC, Baric RS. The species severe acute respiratory syndromerelated coronavirus: classifying 2019-nCoV and naming it SARS-CoV-2. Nat Microbiol 2020;5:536-44.

24 Shang J, Ye G, Shi K, et al. Structural basis of receptor recognition by SARS-CoV-2. Nature 2020;581:221-4.

25 Schultze JL, Aschenbrenner AC. COVID-19 and the human innate immune system. Cell 2021;184:1671-92.

26 Sette A, Crotty S. Adaptive immunity to SARS-CoV-2 and COVID-19. Cell. 2021; $184: 861-80$.

27 Merad M, Martin JC. Pathological inflammation in patients with COVID-19: a key role for monocytes and macrophages. Nat Rev Immunol 2020;20:355-62.

28 Rydyznski Moderbacher C, Ramirez SI, Dan JM, et al. Antigen-Specific adaptive immunity to SARS-CoV-2 in acute COVID-19 and associations with age and disease severity. Cell 2020;183:e19:996-1012.

29 Shah VK, Firmal P, Alam A, et al. Overview of immune response during SARS-CoV-2 infection: lessons from the past. Front Immunol 2020;11:1949. 
30 Saini SK, Hersby DS, Tamhane T, et al. SARS-CoV-2 genome-wide T cell epitope mapping reveals immunodominance and substantial CD8 ${ }^{+} \mathrm{T}$ cell activation in COVID-19 patients. Sci Immunol 2021;6:eabf7550.

31 Bar-Or A, Li R. Cellular immunology of relapsing multiple sclerosis: interactions, checks, and balances. Lancet Neurol 2021;20:470-83.

32 Chowdhury MA, Hossain N, Kashem MA, et al. Immune response in COVID-19: a review. J Infect Public Health 2020;13:1619-29.

33 Vabret N, Britton GJ, Gruber C, et al. Immunology of COVID-19: current state of the science. Immunity 2020;52:910-41.

34 Mehta P, McAuley DF, Brown M, et al. COVID-19: consider cytokine storm syndromes and immunosuppression. Lancet 2020;395:1033-4.

35 Peter AE, Sandeep BV, Rao BG, et al. Calming the storm: natural immunosuppressants as adjuvants to target the cytokine storm in COVID-19. Front Pharmacol 2020;11:583777.
36 Fajgenbaum DC, June CH. Cytokine storm. N Engl J Med Overseas Ed 2020;383:2255-73.

37 Zhou F, Yu T, Du R, et al. Clinical course and risk factors for mortality of adult inpatients with COVID-19 in Wuhan, China: a retrospective cohort study. The Lancet 2020;395:1054-62.

38 Herold T, Jurinovic V, Arnreich C, et al. Elevated levels of IL- 6 and CRP predict the need for mechanical ventilation in COVID-19. Journal of Allergy and Clinical Immunology 2020;146:128-36.

39 Thwaites RS, Sanchez Sevilla Uruchurtu A, Siggins MK, et al. Inflammatory profiles across the spectrum of disease reveal a distinct role for GM-CSF in severe COVID-19. Sci Immunol 2021;6:eabg9873.

40 Salama C, Han J, Yau L, et al. Tocilizumab in patients hospitalized with Covid-19 pneumonia. N Engl J Med Overseas Ed 2021;384:20-30. 